\section{Telemedicina en otorrinolaringología}

\section{Telemedicine in otorhinolaryngology}

\author{
Paul Délano R. ${ }^{1}$
}

\author{
${ }^{1}$ Editor. Revista de Otorrinolaringología y Cirugía de \\ Cabeza y Cuello. Departamento Otorrinolaringología \\ Hospital Clínico Universidad de Chile. Santiago, Chile. \\ Correspondencia: \\ Dr. Carlos Lorca Tobar, 999 \\ Servicio Otorrinolaringología. Hospital Clínico Universidad \\ de Chile. Independencia. Santiago, Chile. \\ Email: pdelano@med.uchile.cl
}

Antes de la pandemia del SARS-CoV-2 la telemedicina ya se utilizaba en forma rutinaria en disciplinas como radiología o cardiología, siendo una herramienta que facilita el diagnóstico de pacientes en lugares remotos, que tienen poca accesibilidad a atención médica especializada. La pandemia del COVID-19 nos ha obligado a acelerar el desarrollo de plataformas y tecnologías para la atención médica a distancia. Durante esta pandemia, el Ministerio de Salud de Chile avaló la telemedicina para diversas especialidades médicas a través del pago de prestaciones virtuales, pero, lamentablemente, otorrinolaringología no fue incluida en este grupo de especialidades.

En este número de la revista se presentan dos trabajos de investigación realizados durante la pandemia COVID-19 en Chile y Paraguay, en los cuales se muestran los beneficios de la telemedicina en otorrinolaringología. En el primer trabajo, García-Huidobro y cols. muestran que los pacientes y profesionales tienen un alto grado de satisfacción con el uso de la telemedicina en el Hospital Sótero del Río en Santiago de Chile. En el segundo trabajo, Mena y cols. demuestran que el uso de telemedicina en otorrinolaringología disminuyó en más de un $70 \%$ la necesidad de consultas presenciales durante la pandemia COVID-19 en el Hospital de Clínicas de San Lorenzo, Paraguay. Estos trabajos y la literatura internacional demuestran la utilidad de la atención virtual en otorrinolaringología, incluyendo primeras consultas en audiología, otoneurología, y alergias entre otras patologías. La telemedicina puede ser también una herramienta importante para controles médicos o evaluación de exámenes en una segunda consulta y para el triage de patologías que necesitan de evaluación presencial en otorrinolaringología, como tumores o colesteatomas. La utilidad de la telemedicina en otorrinolaringología es evidente, por lo que nuestras autoridades debieran considerarla como una prestación subsidiada.

Respecto a nuestra revista, en el número de septiembre del 2020 renovamos su imagen y diagramación. La imagen de la portada de la revista irá cambiando en cada número, la que será elegida como la figura más destacada de los artículos publicados. Los criterios para seleccionar a la imagen de la portada serán: originalidad, importancia científica para la disciplina y calidad artística. Otro cambio es que hemos vuelto a incorporar el color a las figuras de manera gratuita para todos los autores, tanto en la versión impresa como en la digital. De esta forma, invito a nuestros autores a enviar figuras originales en colores y de la más alta calidad, que las hagan candidatas a ser portada de nuestra revista.

La portada del número de diciembre del 2020 corresponde a una imagen ecográfica de cuello publicada en el artículo de Segall y cols., donde se revisa la utilidad del ultrasonido realizado por el otorrinolaringólogo. En este número, destacó también el trabajo de investigación de Valdés y cols. que valida la encuesta de calidad de vida SNOT-22 para rinosinusitis crónica en la población chilena y el caso clínico publicado por Goycoolea y cols. donde se presenta la colocación exitosa un implante coclear en una paciente de 98 años. 